Cita bibliográfica: Rato Martín, H., y Fernández Cuesta, G. (2018). El proyecto de reforma y ensanche de la villa de Llanes de 1905. Boletín de la Asociación de Geógrafos Españoles, 76, 414-429. doi: $10.21138 /$ bage. 2528

\title{
El proyecto de reforma y ensanche de la villa de Llanes de 1905
}

The reform and urban extension of the village of Llanes' project in 1905

\author{
Héctor Rato Martín \\ hector.rato@gmail.com \\ Gaspar Fernández Cuesta \\ gaspar@uniovi.es \\ Departamento de Geografía \\ Universidad de Oviedo (España)
}

\section{Resumen}

La redacción de proyectos de ensanche a partir de mediados del siglo XIX fue un hecho habitual en las principales ciudades españolas, pero también tuvo su plasmación en algunos núcleos de menor jerarquía, como en la villa de Llanes. En el presente artículo se presenta el proyecto de ensanche y reforma interior de la población llanisca realizado por el ingeniero Alberto Corral a principios del siglo pasado, describiendo las características del mismo y el proceso de elaboración, así como su legado urbanístico en la villa actual.

Palabras clave: villa; ensanche; urbanismo; Asturias.

\begin{abstract}
The drafting of urban extension projects has typically been done in the largest Spanish cities since the middle of the XIXth century. However it was also a present in some towns despite lower status, for example, the village of Llanes. The urban extension and internal reform of Llanes project, designed by the engineer Alberto Corral at the beginning of the last century, is featured in this
\end{abstract}


article. Characteristics of the project and the working process are described, along with the report of its urban legacy in the current village.

Key words: village; urban extension; urban planning; Asturias.

Agradecimientos: Este artículo es resultado de la concesión de una beca predoctoral para la Formación en Investigación y Docencia, Ficyt, Gobierno del Principado de Asturias, expediente. BP12-125.

\section{El marco legal}

Las ciudades españolas, que habían experimentado pocos cambios en su morfología durante la Edad Media y la Edad Moderna, dieron muestras de la incapacidad de la estructura urbana heredada para hacer frente a las nuevas necesidades económicas y sociales surgidas a partir de mediados del siglo XIX. Necesidades ligadas a la industrialización y a la aparición de una burguesía enriquecida con las actividades fabriles, fenómenos ambos que forzaban un cambio en el modelo de ciudad imperante hasta entonces. El núcleo heredado debía de ser transformado a fin de adaptar su tortuosa trama viaria a los mayores tráficos surgidos con la instalación de fábricas y la llegada del ferrocarril; la burguesía, nueva clase dominante, demandaba edificios residenciales de mayor tamaño y mejores condiciones de salubridad y las murallas, una vez que habían quedado desposeídas de su función, constituían un límite físico para la expansión. Este crecimiento espacial, por otra parte, también requería de una serie de estándares de rectitud y ancho, de la dotación de plazas y equipamientos..., frente al crecimiento orgánico que había presidido la edificación de viviendas durante el Antiguo Régimen.

A fin de regular la transformación de la ciudad histórica y de ordenar la expansión futura de la población, el Estado adquirió en estos años competencias normativas en materia urbanística, anteriormente en manos de los ayuntamientos, que eran los encargados de redactar las ordenanzas municipales y las normas de policía urbana particulares (De Terán, 1999, p. 52). Los instrumentos empleados por el gobierno central para dirigir y ejecutar dichas actuaciones fueron las leyes de reforma interior y las leyes de ensanche. ${ }^{1}$

A lo largo de la segunda mitad del siglo XIX entraron en vigor de manera sucesiva tres leyes de ensanche (1864, 1876 y 1892), con sus respectivos reglamentos, en las que se incluían una serie de artículos encargados de establecer "los deberes de los propietarios del suelo y de la

1 Una detallada recopilación de las principales figuras normativas urbanísticas del siglo XIX y la primera mitad del XX es Bassols Coma, M. (1973). Génesis y evolución del derecho urbanístico español (1812-1956). 
Administración, fórmulas para la cesión de viales, mecanismos de reparcelación o las cargas y beneficios que correspondían a los propietarios del suelo" (Fernández, 2015, p. 11).

A partir de estas figuras legislativas muchas ciudades españolas aprobaron en esa época, extendida hasta las primeras décadas del siglo XX, proyectos de expansión que fijaban la forma en la que se expandiría su plano. Generalmente fueron las ciudades con mayor número de habitantes y más dinamismo demográfico las que impulsaron la aprobación de ensanches, aunque también hubo otras de inferior jerarquía que redactaron (con mayor o menor éxito) documentos de estas características. $^{2}$ En Asturias tenemos ejemplos de proyectos de ensanches menores en algunas localidades de las cuencas mineras ${ }^{3}$ que, pese a su inferior tamaño, copaban buena parte de la actividad económica regional; también en algunas villas costeras, como Ribadesella o Llanes. ${ }^{4}$ En la población llanisca la actividad industrial era residual (Canella, 1994, pp. 37-38) y la pesca y el tráfico marítimo habían decaído (Canella, 1994, pp. 16-18) respecto a los niveles alcanzados durante los siglos XVI y XVII, cuando llegó a ser el segundo puerto en importancia de la región (Uria, 2004, p. 67). Sin embargo, los capitales retornados de América por los emigrantes que regresaban de su estancia en tierras de ultramar y las remesas enviadas por la importante colonia llanisca asentada en el continente americano sirvieron para financiar importantes mejoras urbanas en todo el municipio (Morales, 1999, pp. 63-81)

\section{Los precedentes del proyecto de ensanche}

La necesidad de disponer de un proyecto de ensanche que señalase las alineaciones a las que deberían de ceñirse las nuevas construcciones levantadas en la villa se hizo evidente en las últimas décadas del siglo XIX. El aumento de la población, unido a la escasez de solares en el casco urbano y a la necesidad de planificar la futura expansión de la villa, llevaron al ayuntamiento a gestionar la elaboración de un documento cartográfico director del desarrollo espacial. Sin embargo, las primeras tentativas no pasaron de meros estudios o trámites que no llegaron a materializarse en un plano a causa de razones de distinta índole, la mayoría económicas.

Hubo que esperar al año 1886 para que viese la luz el primer proyecto de ensanche de Llanes. Su autor fue Tomás Álvarez Diez Morán, arquitecto e ingeniero que ejercía como catedrático en el colegio de segunda enseñanza de la villa. El ayuntamiento aceptó el ofrecimiento de Tomás Álvarez

2 Son los conocidos generalmente como "ensanches menores". Varios ejemplos de ensanches menores en poblaciones catalanas han sido estudiados por Julio Esteban Noguera en Esteban i Noguera, J. (1976). Los ensanches menores en la región de Barcelona (II).

3 El caso de Langreo es bien conocido gracias a Fernández García, A. (1980). Langreo: industria, población y desarrollo urbano.

4 La evolución económica, demográfica y funcional de varias localidades de la costa asturiana a partir de mediados del siglo XIX ha sido analizada en profundidad por Emilio Murcia en Murcia Navarro, E. (1981). Las villas costeras en el sistema urbano asturiano. 
para levantar, a cambio de 2500 pesetas, el primer plano geométrico de la villa, que incluía además una propuesta de nuevas alineaciones para el casco urbano y un proyecto de ensanche. ${ }^{5}$ Las zonas de reforma interior y expansión urbana fueron propuestas por una delegación del consistorio, que se encargó de indicar al ingeniero los puntos más adecuados para tal fin. ${ }^{6} \mathrm{El}$ crecimiento de la villa seguiría como eje director la carretera general, generándose así dos nuevas zonas de trama ortogonal en las salidas Noroeste y Sur de la villa. En este segundo espacio, de mayor extensión, se construirían también un mercado de pescado, una alhóndiga, una plaza de abastos y un mercado de ganado. ${ }^{7}$

Una vez finalizado, el proyecto contó con el visto bueno de las comisiones municipales de urbanismo y ornato y, tal y como establecía la normativa, fue expuesto al público durante veinte días. ${ }^{8}$ En esta fase, varios propietarios mostraron su disconformidad con aquellas actuaciones planteadas que implicaban la expropiación de algunas de sus fincas urbanas, al considerar que dichas obras no eran imprescindibles. Señalaban, además, que el plano carecía de información sobre las rasantes de las calles y que no se habían seguido los preceptos de la Ley de Ensanche de 1876 y su reglamento, sino las prescripciones de normativas anteriores. ${ }^{9}$ Las alegaciones fueron tenidas en cuenta por el ayuntamiento y enviadas al Arquitecto provincial ${ }^{10}$ pero, con anterioridad a que éste dictase una resolución al respecto, Tomás Álvarez retiró el proyecto por la oposición mostrada por la población llanisca. ${ }^{11}$

En los años inmediatamente posteriores a la fallida aprobación del ensanche de 1886, el interés municipal por disponer de un documento que dirigiese de manera unitaria el urbanismo local pareció decaer. El fracaso de la iniciativa anterior y los altos costes y las dificultades administrativas que implicaban la realización de uno nuevo hicieron que, según el consejo del arquitecto provincial, se siguiesen utilizando planes parciales de alineaciones y rasantes para cada una de las actuaciones concretas. $^{12}$ Sin embargo, la necesidad de un plan urbanístico global era evidente, puesto que la

5 Archivo Municipal de Llanes (AHMLL). Libros de actas del concejo, libro n²9, sesión del día 14 de junio de 1884, acuerdo $\mathrm{n}^{\circ} 12$.

6 AHMLL. Libros de actas del concejo, libro n² 29, sesión del día 25 de abril de 1885, acuerdo n 21 y sesión del día 24 de octubre de 1885, acuerdo n 8.

7 AMLL. Expediente 467-13. Memorias correspondientes al plano de la villa de Llanes, años 1885-1886. Desafortunadamente no se conserva la cartografía del proyecto, por lo que desconocemos las características concretas de la nueva trama urbana ideada.

8 AHMLL. Libros de actas del concejo, libro n 30, sesión del día 1 de mayo de 1886, acuerdo n 9.

9 AHMLL. Libros de actas del concejo, libro n 30, sesión del día 19 de junio de 1886, acuerdo n 1 y sesión del día 26 de junio de 1886, acuerdo n 1.

10 AHMLL. Libros de actas del concejo, libro n³0, sesión del día 29 de enero de 1887, acuerdos n 2 y 3.

11 AHMLL. Libros de actas del concejo, libro n 30, sesión del día 29 de enero de 1887, acuerdo n 4.

12 AHMLL. Libros de actas del concejo, libro n³2, sesión del día 24 de octubre de 1891, acuerdo n 3. 
iniciativa particular, principal promotora del trazado de calles y construcción de viviendas, lo hacía sin seguir, en líneas generales, orden alguno. ${ }^{13}$

Figura 1. Llanes en 1905

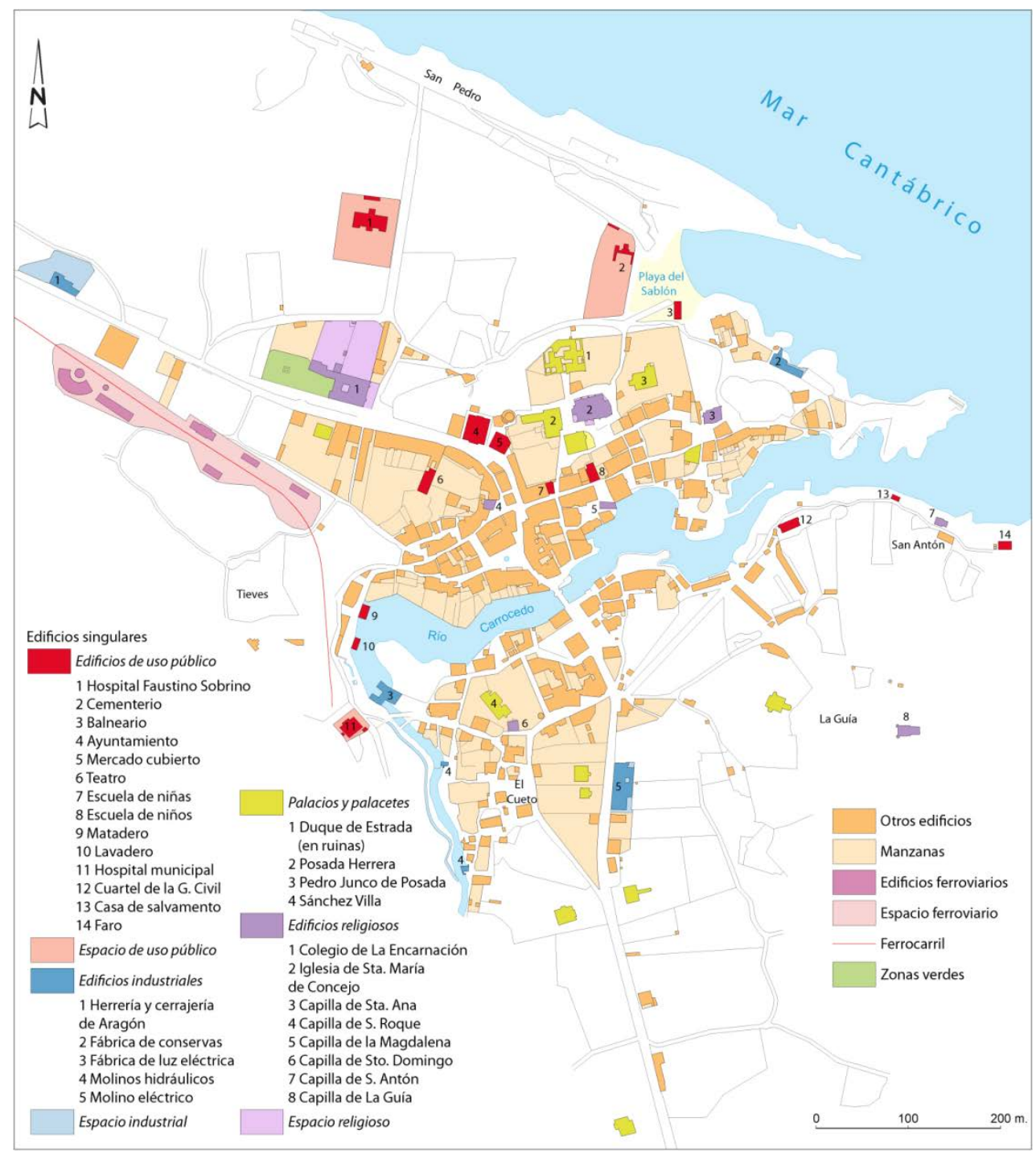

Leyenda: La villa todavía mantenía a principios del siglo XX buena parte de la trama urbana heredada del antiguo régimen. Las nuevas viviendas promovidas por la burguesía a finales del XIX se localizaban preferentemente a lo largo de las carreteras de salida hacia Oviedo y Santander, por lo que su construcción no implicó la apertura de nuevas calles. Algunos equipamientos, como el balneario, el mercado cubierto, el hospital municipal o las nuevas fábricas y la llegada del ferrocarril simbolizan el auge de la villa tras más de un siglo de estancamiento

Fuente: elaboración propia a partir del Plano de la villa de Llanes levantando por el Ingeniero de Caminos, Canales y Puertos D. Alberto Corral (1902) y del Plano general de Llanes (mayo 1905)

13 Especialmente problemático debía de ser el caso de las nuevas viviendas levantadas en los márgenes de la carretera de Santander al Sur de la villa, en La Concepción, puesto que la opinión pública recogía la falta de aceras, la irregular línea de fachadas que formaban estas construcciones y la ausencia de calles transversales. El Oriente de Asturias, 30 de agosto de 1896 y 14 de febrero de 1897. En otros puntos de la villa, como en la calle Calzada, las edificaciones de nueva planta también debían de carecer de alineación (Morales, 1993, pp. 22 y 209). 


\section{La gestación del proyecto}

En vista de los sucesivos fracasos para elaborar el plano geométrico y de ensanche por iniciativa pública, en el año 1899 Toribio García Álvarez y sus hermanos se ofrecieron a pagar por su cuenta un plano de población. El coste de realización de este plano, que serviría para conocer el estado de la villa y se utilizaría como base para la elaboración de un proyecto que dirigiese las reformas interiores y la expansión urbana, se estimaba en unas 3000 pesetas. ${ }^{14}$ El ayuntamiento aceptó el generoso ofrecimiento particular y contactó con Alberto Corral, ingeniero burgalés afincado en Santander (Sazatornil, 1996, p. 286), para que se hiciese cargo de los trabajos. Tras acceder éste a la tarea confiada se trasladó a Llanes en octubre del año 1900 para dar inicio al levantamiento de la cartografía. ${ }^{15}$

Alberto Corral trabajó aproximadamente durante año y medio en la realización del plano, que entregó al consistorio en mayo de $1902 .{ }^{16}$ Compaginó esta labor con la realización de otros trabajos que le fueron encomendados aprovechando su presencia en la villa, como la terminación del proyecto del nuevo cementerio o la mejora de los paseos de San Pedro y de Posada Herrera. ${ }^{17}$ La cartografía se hizo a escala 1:400, con curvas de nivel cada metro, y en ella se representaba la localidad y su entorno prestando especial atención al parcelario y al caserío. Tras el levantamiento del plano, los hermanos García Álvarez pagaron al ayuntamiento los costes del documento, que finalmente ascendieron a 5000 pesetas. $^{18}$

Una vez que por fin se disponía de un documento cartográfico que mostraba con precisión el estado de la trama urbana, se estaba en condiciones de realizar el proyecto de ensanche y reforma interior. En esta ocasión el ayuntamiento se comprometió a costearlo por su cuenta, presupuestando en 5000 pesetas su redacción, que también fue encargada a Alberto Corral. ${ }^{19}$ La adjudicación se hizo en febrero de 1903 y el ingeniero trabajó durante dos años en la confección del proyecto, que entregó en junio de $1905 .{ }^{20}$ Como ocurriese con anterioridad, de manera simultánea se hizo cargo de otras obras, como fue la realización del proyecto general de alcantarillado de la localidad. ${ }^{21}$

14 AHMLL. Libros de actas del concejo, libro n³6, sesión del día 8 de julio de 1899, acuerdo n 5.

15 El Oriente de Asturias, 28 de octubre de 1900.

16 AHMLL. Libros de actas del concejo, libro n 37, sesión del día 24 de mayo de 1902, acuerdo n 6.

17 AHMLL. Libros de actas del concejo, libro n³6, sesión del día 1 de diciembre de 1900, acuerdo nº 6.

18 AHMLL. Libros de actas del concejo, libro n 38, sesión del día 4 de abril de 1903, acuerdo nº 10.

19 AHMLL. Libros de actas del concejo, libro n³8, sesión del día 14-2-1903, acuerdo nº 15.

20 AHMLL. Libros de actas del concejo, libro n 39, sesión del día 3 de junio de 1905, acuerdo n 17.

21 AHMLL. Libros de actas del concejo, libro n³ 38, sesión del día 10 de diciembre de 1904, acuerdo nº 16. 
Tras recibir el expediente de reforma y ensanche el ayuntamiento inició los trámites necesarios para su aprobación por la Superioridad, ${ }^{22}$ pero, por lo que indican algunas fuentes posteriores, la falta de algunos detalles burocráticos impidió que contase con el visto bueno gubernamental. ${ }^{23}$ Las gestiones debieron de frenarse en ese momento y el expediente nunca debió de entró en vigor, puesto que en la década de 1920 se reclamaba desde la opinión pública la necesidad de recuperar el proyecto para ordenar el desarrollo urbano local, carente de documento director. ${ }^{24}$ Por lo que respecta al plano topográfico de 1902, sabemos que a principios de siglo fue aprobado por parte municipal y que fue expuesto al público durante veinte días, ${ }^{25}$ pero desconocemos si posteriormente fue enviado a instancias superiores para su aprobación definitiva.

\section{Contenido del proyecto ${ }^{26}$}

El Proyecto de reforma y ensanche de la villa de Llanes de 1905 constaba de una memoria, un plano general a escala 1:2000, diez planos de detalle a escala 1:400 y varios perfiles longitudinales y transversales. El grueso de la documentación se conserva actualmente en el Archivo Histórico de Llanes, excepto el plano general y los de detalle, que lamentablemente han desaparecido de la carpeta del expediente. Afortunadamente, en el fondo particular Remigio Fernández del Archivo Municipal de Ribadedeva se custodia un cianotipo del plano general, que nos ha servido como base para la realización del que incorporamos en este artículo.

La memoria consta de cuatro apartados: "Generalidades sobre el trazado de poblaciones", "Descripción de Llanes", "Descripción del proyecto y su justificación" y "Consecuencias del proyecto y su realización". En la primera parte se desarrolla un modelo teórico acerca de la forma en la que ha de actuarse en los núcleos urbanos a fin de corregir los problemas de la ciudad heredada y de ordenar el futuro crecimiento de la población. Así, se van dando detalles sobre el emplazamiento ideal de las zonas de ensanche, el tipo de trama más adecuado, los distintos usos del suelo idóneos para cada zona, las características de las calles y el mobiliario urbano, la localización de los edificios públicos, etc. Esta introducción es de gran utilidad para comprender la solución que el autor eligió para Llanes.

A continuación, la memoria trata el caso concreto de la villa. En primer lugar se relata someramente cómo fue la historia de la localidad, utilizando como fuente el libro de Fermín Canella Historia de Llanes y su concejo, y se mencionan algunos datos sobre la topografía, el clima, las

22 AHMLL. Libros de actas del concejo, libro n³9, sesión del día 10 de junio de 1905, acuerdo n².

23 El Pueblo, 19 de diciembre de 1926.

24 El Oriente de Asturias, 23 de abril de 1927, 8 de octubre de 1927 y 29 de octubre de 1927.

25 AHMLL. Libros de actas del concejo, libro n 39, sesión del día 10 de junio de 1905, acuerdo n 2.

26 AHMLL. Expediente 467-2. Proyecto de reforma y ensanche de la villa de Llanes, 1905. 
comunicaciones, la economía o la demografía locales. Posteriormente, el autor pasa a explicar de manera extensa su propuesta de reforma del casco urbano y las nuevas alineaciones que diseña para dirigir la expansión urbana.

Figura 2. Plano del Proyecto de reforma y ensanche de la villa de Llanes (1905)

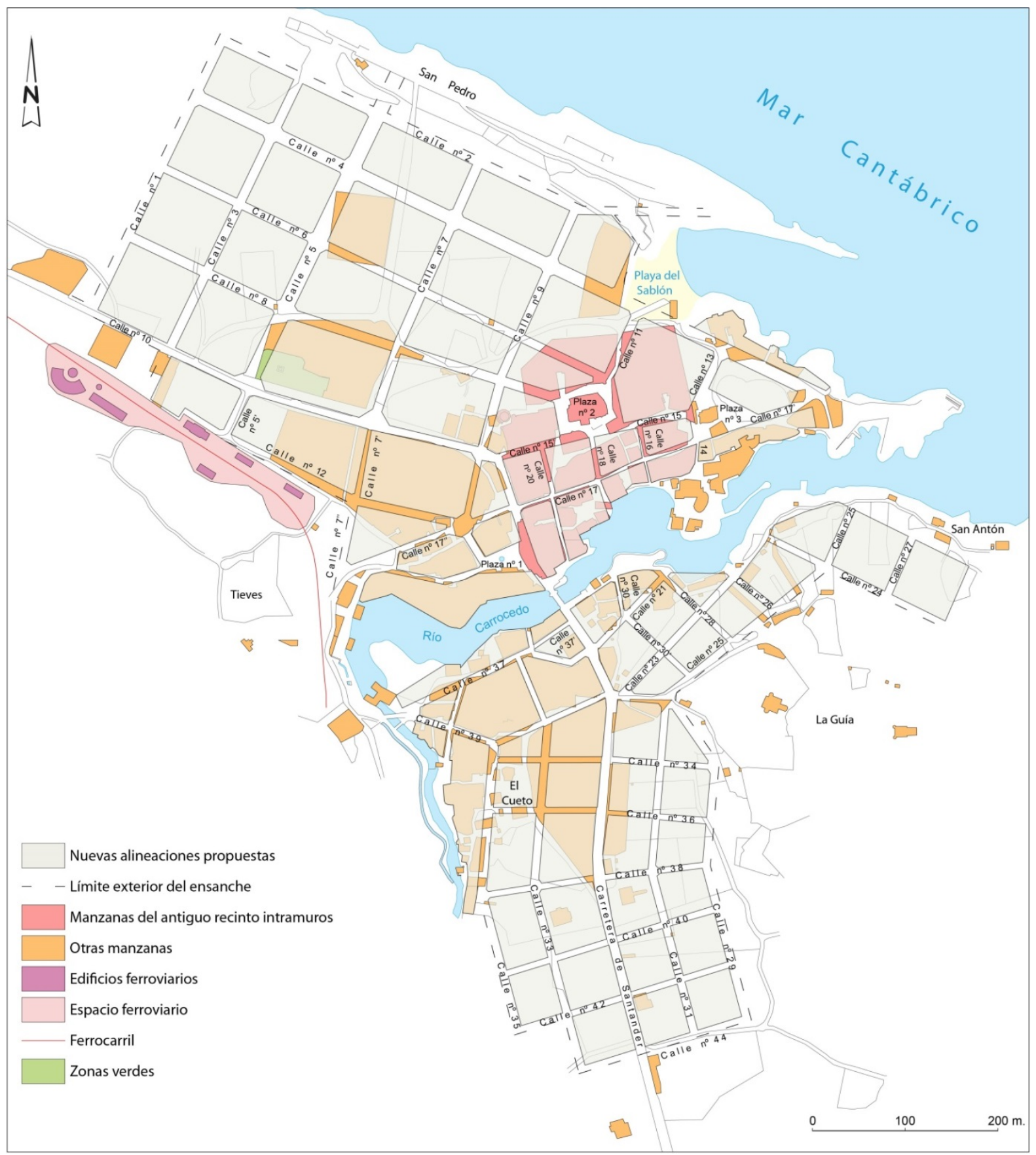

Leyenda: Alberto Corral ideó un ensanche en el que se pueden diferenciar tres espacios con características propias, tanto por la forma de su trama como por los usos asignados. Eran los barrios de Posada Herrera, la Portilla y San Antón, localizados al noroeste, sur y noreste de la localidad, respectivamente. También planteaba una serie de actuaciones en la ciudad heredada fin de adaptarla a los nuevos gustos y necesidades de la época.

Fuente: elaboración propia a partir del Plano de la villa de Llanes levantando por el Ingeniero de Caminos, Canales y Puertos D. Alberto Corral (1902) y del Plano general de Llanes (mayo 1905) 


\subsection{La reforma interior}

El proyecto de reforma interior no planteaba, en líneas generales, una alteración sustancial de la red viaria preexistente. El rumbo quebrado de buena parte de los viales, herencia directa en muchos casos de la Edad Media, y la aglomeración de inmuebles en algunas partes del casco urbano hacían poco factible una regularización del plano sin que ello implicase el derribo de casi todas las edificaciones. En consecuencia, las actuaciones planificadas consistirían, en su mayoría, en la alineación, rectificación y simplificación del trazado de las calles más importantes (como la calle Mayor, la calle Calzada o la carretera general), manteniendo los edificios de mayor valor histórico o reciente construcción.

\section{Figura 3. Vista parcial de Llanes tomada desde Tieves. Postal editada por la Librería General (Santander)}

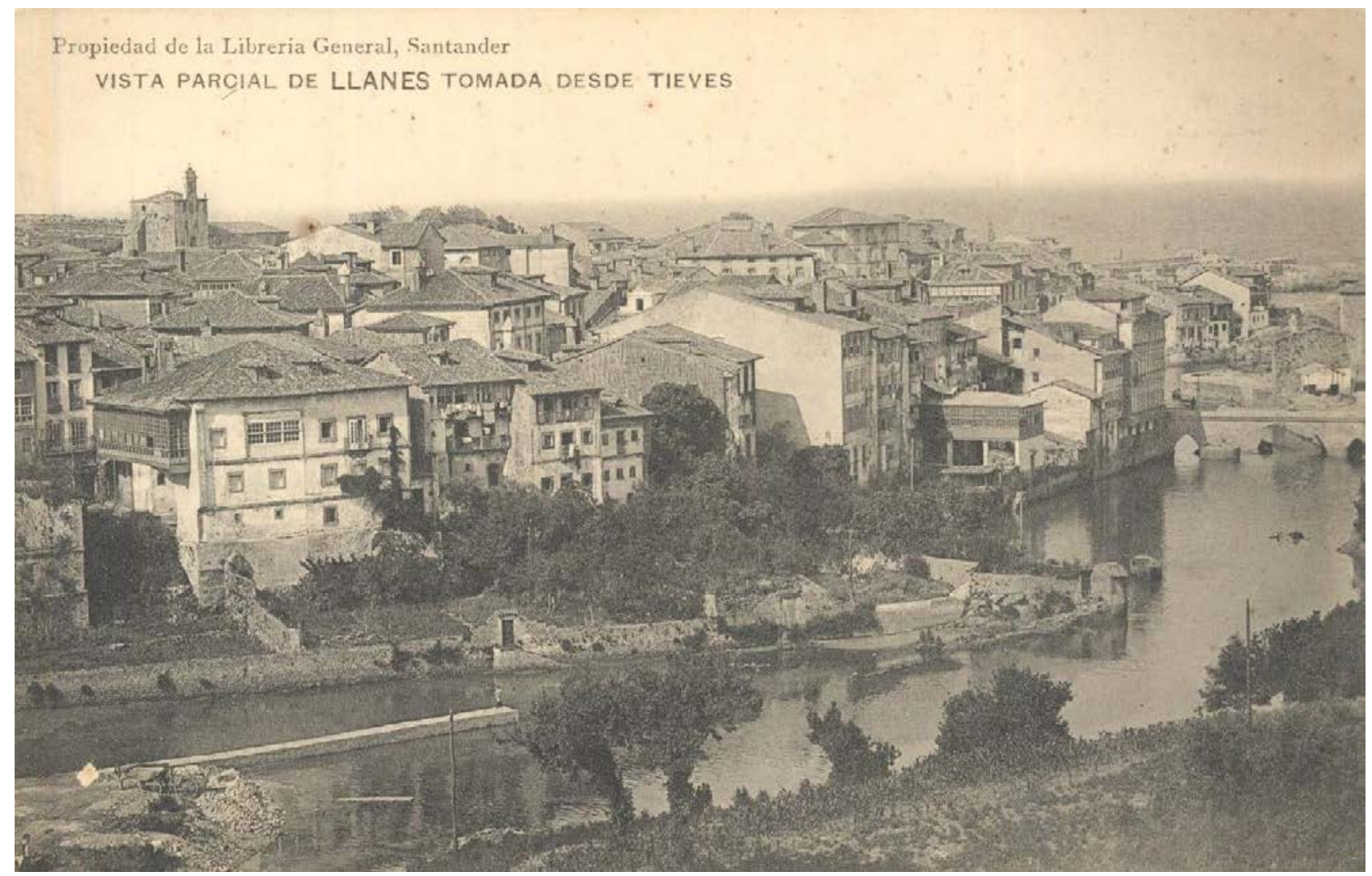

Leyenda: Debido a lo abigarrado de la trama urbana y a los escasos espacios libres existentes entre las edificaciones, las propuestas de Alberto Corral para la reforma de la ciudad histórica no suponían una alteración radical del viario. Consistirían, sobre todo, en la prolongación y alineación de las calles principales.

Fuente: Museo de Artes y Costumbres Populares de Sevilla

Otra de las correcciones propuestas sería la apertura de nuevos viales dentro del casco urbano para facilitar el tráfico hacia algunas de las zonas en las que se desarrollaría mayor actividad, como la estación o el muelle de Santiago, o para desahogar de circulación la carretera general. En otras 
ocasiones, el trazado de nuevas calles respondía a la necesidad de engarzar el núcleo consolidado con las futuras zonas de ensanche.

Las operaciones urbanísticas de mayor transcendencia sobre el plano y que implicaban un notable número de derribos se realizarían en aquellos espacios en los que el caserío era más humilde y, consecuentemente, menos costosas las expropiaciones. Así ocurría en el barrio del Cueto, en el que el escaso valor de las viviendas y lo abigarrado de la trama llevaron al ingeniero a diseñar una reforma completa del viario gracias a la apertura de varias calles de trazado rectilíneo. Otras actuaciones a gran escala tendrían como finalidad la ampliación de varias plazas, como la de la Iglesia, la plaza de Santa Ana o la plaza Mayor; ésta última como resultado del ensanche de la calle Mercaderes en las proximidades del puente.

El espacio inmediato al puerto quedaría al margen de cualquier actuación urbanística, salvo en la parte más próxima al puente. Alberto Corral consideraba que la reorganización de esta zona debería realizarse de manera simultánea a las obras de mejora de las instalaciones portuarias, por lo que resultaría más apropiado incluir su diseño en el proyecto de ampliación de los muelles.

Figura 4. Llanes: vista parcial del Ribero. Postal editada por la Librería General (Santander)

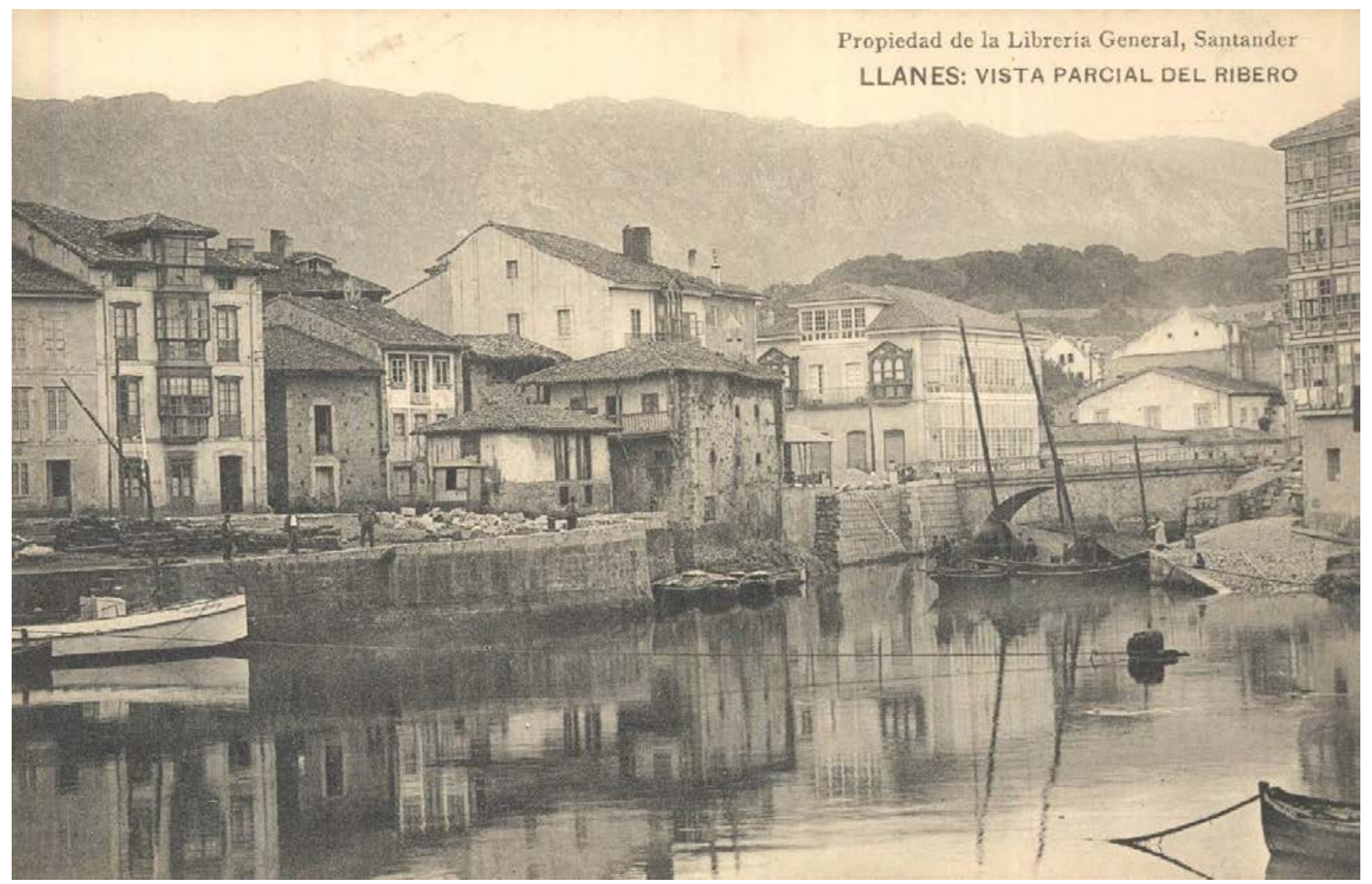

Leyenda: El proyecto de ensanche no planteaba grandes modificaciones en las márgenes de la ría, desde el puente hasta la desembocadura, pues la urbanización de este espacio debería de concretarse una vez que se realizasen las obras interiores del puerto, aún sin definir. Las dos viviendas situadas en la margen izquierda, junto al Carrocedo, desaparecerían con posterioridad como consecuencia de los trabajos de prolongación del muelle de las Barqueras.

Fuente: Museo de Artes y Costumbres Populares de Sevilla 
Junto a las medidas encargadas para corregir las deficiencias en la trama de la ciudad heredada, también se proponía la dotación de nuevos edificios públicos. La iglesia, el ayuntamiento y el juzgado mantendrían su ubicación, por sus buenas condiciones y óptimas comunicaciones. El hospital municipal y el nuevo cementerio tampoco variarían su emplazamiento, al estar construidos en lugares adecuados para sus necesidades, pero no ocurría lo mismo con el mercado, cuyas pequeñas dimensiones y mal estado de conservación invitaban al levantamiento de un nuevo edificio de mayor tamaño más próximo a la estación y a la plaza del mercado. En último término, todas las medidas propuestas tendrían como objetivo la especialización comercial del núcleo urbano.

\subsection{El ensanche}

La trama diseñada por el ingeniero para dirigir la futura expansión de la población se desarrollaría sobre los escasos terrenos llanos presentes en las inmediaciones de la villa, al constituir el mar Cantábrico, el río Carrocedo y las colinas que circundaban al núcleo urbano límites infranqueables para el crecimiento espacial. Consecuentemente, el ensanche se proyectaría sobre tres espacios situados al Noroeste (barrio de Posada Herrera), al Sur (barrio de la Portilla) y al Noreste (barrio de San Antón) de la localidad. La apertura de nuevos viales en estas zonas se haría atendiendo a la inclinación, procurando que fuese lo menor posible, y a las condiciones ambientales, considerando los vientos dominantes y la exposición solar.

El barrio de Posada Herrera ocuparía los terrenos llanos existentes entre el Paseo de San Pedro, el Sablón y la salida hacia Oviedo por la carretera general. En su mayor parte ocuparía fincas rústicas que no suponían obstáculo alguno para la apertura de nuevas calles, pero en la zona también existían algunas edificaciones que dificultaban el trazado de los viales. Los más importantes por sus dimensiones eran el hospital particular Faustino Sobrino y el antiguo cementerio, cuyo derribo se consideraba justificado por la mala ubicación del primero y la obsolescencia del segundo, que sería sustituido por un nuevo camposanto ya casi terminado. Sí se respetaría el edificio del colegio de la Encarnación, aunque una pequeña parte del mismo sería expropiada para ampliar el paseo de Posada Herrera.

La ocupación de esta zona del ensanche se haría mediante una trama ortogonal formada por una serie de calles paralelas y perpendiculares a la carretera general, que actuaría como eje director de la cuadrícula. Las únicas alteraciones a la trama ortogonal serían consecuencia de la variación del trazado de calles que funcionasen como engarce con el casco antiguo o cuya apertura tuviese como objetivo el acceso a determinados espacios como el Paseo de San Pedro.

La orientación de los viales permitía que las edificaciones levantadas en sus márgenes recibieran en sus cuatro fachadas la luz solar durante todo el año y que la exposición a los vientos dominantes no fuese directa. En consecuencia, las condiciones eran las más adecuadas para construir viviendas 
destinadas a la burguesía; este hecho también se tuvo en cuenta para diseñar el tamaño de las manzanas, las mayores de todo el proyecto, a fin de poder construir en ellas casas unifamiliares con jardín.

El ancho estándar de las nuevas calles sería de 10 metros con la salvedad de las calles números 4 y 5, dos bulevares arbolados de 15 metros de ancho paralelos y perpendiculares a la carretera respectivamente. El cruce de las calles con estos bulevares se haría mediante chaflanes.

Figura 5. Llanes: jardines y estatua de Posada Herrera.

Postal editada por la Librería General (Santander)

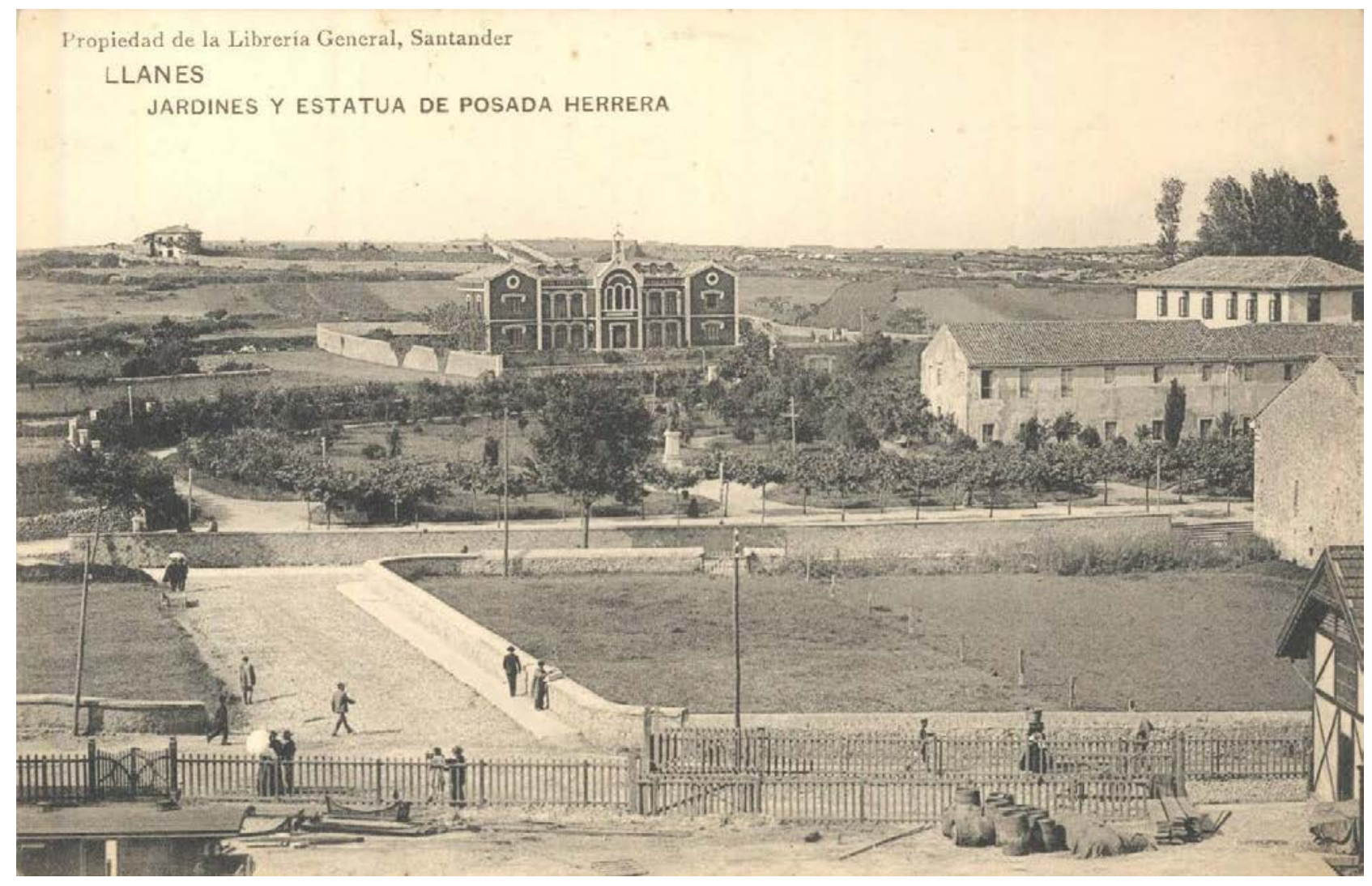

Leyenda: El barrio de Posada Herrera se localizaría al oeste de la población, entre el paseo de San Pedro y la carretera de Oviedo. Aunque fundamentalmente se trataba de terrenos baldíos, lo que facilitaba la urbanización, en la zona también existían algunas edificaciones de gran tamaño, como el colegio de la Encarnación (en el centro de la imagen, a la derecha) y el hospital Faustino Sobrino (al fondo), que se preveía que fuera expropiado y derribado.

Fuente: Museo de Artes y Costumbres Populares de Sevilla

El barrio de la Portilla estaría emplazado al Sur de la población, entre el río Carrocedo y las elevaciones de La Guía y La Galea. Siguiendo como eje director la carretera a Santander se proponía un sistema de calles paralelas y perpendiculares de 7 y 9 metros de ancho. Resultado de la intersección de los viales era una trama de marcado carácter ortogonal, cuyas únicas variaciones eran consecuencia de la adaptación al trazado de la carretera. El uso previsto para esta zona era el industrial. 
Figura 6. Llanes: puente y calle de Pidal. Postal editada por la Librería General (Santander)

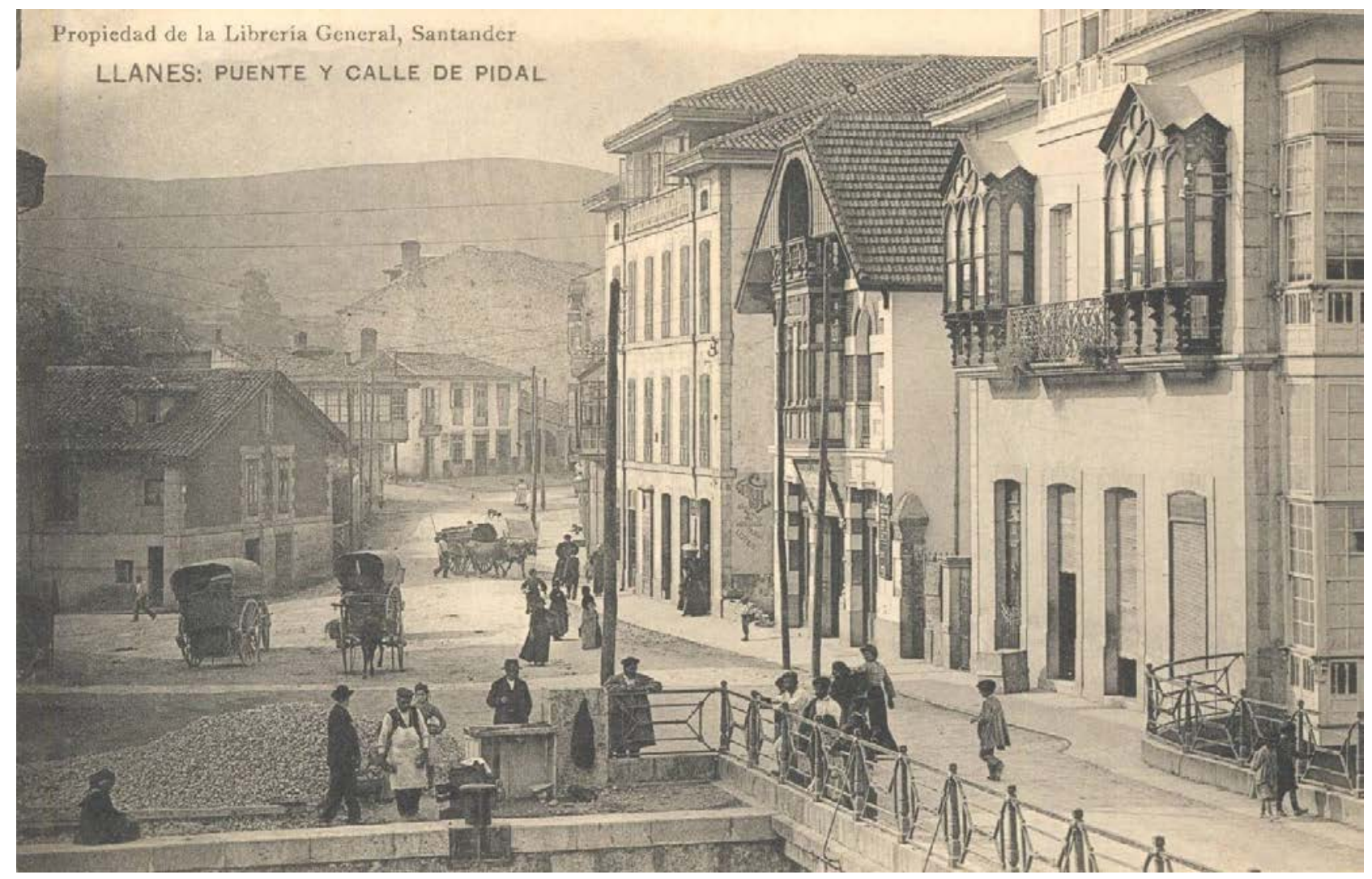

Leyenda: Al sur del puente, la carretera de Santander actuaría como eje director de la trama del barrio de la Portilla. Para ello, planteaban uniformar su anchura a lo largo de todo su recorrido, así como utilizarlo de punto de partida para la apertura de algunas calles que debían de mejorar los accesos a San Antón, la Calzada y el Cueto.

Fuente: Museo de Artes y Costumbres Populares de Sevilla

El barrio de San Antón ocuparía la estrecha franja de terreno llano existente entre el mar y el alto de La Guía, al Noreste del núcleo urbano. Debido a las limitaciones espaciales, únicamente se planteaba la apertura de tres nuevas calles, de cuya intersección resultarían tres manzanas dispuestas en hilera en paralelo a la costa. Aunque por su orientación, similar a la del barrio de Posada Herrera, las manzanas de este barrio eran muy adecuadas para uso residencial, la cercanía del puerto motivó su elección como zona industrial. Para los terrenos colindantes con el mar no se planificaba actuación alguna, al igual que en la otra orilla, pues como ya hemos dicho se creía que su ocupación tendría que concretarse en el proyecto de mejora del puerto.

Junto a la propuesta de ensanche, el ingeniero también recomendaba dos obras de infraestructura que serían de gran utilidad. La primera era un puente giratorio en la bocana del puerto y la segunda el cubrimiento del río Carrocedo entre el puente de la carretera y el del Cagalín, en el Cueto.

La ejecución de un proyecto tan ambicioso no hacía recomendable su realización completa de manera inmediata debido a los altos costes que entrañaría para los presupuestos municipales, por 
lo que las actuaciones propuestas se harían de manera progresiva. Una vez incorporados los nuevos terrenos al núcleo urbano, éste tendría capacidad para albergar a una población superior a los 14000 habitantes, que es la que se creía que podría alcanzar Llanes en el año 1966 si se mantuviese el incremento de población de ese momento.

\section{El legado del proyecto}

Como hemos señalado, parece ser que el Proyecto de reforma y ensanche de la villa de Llanes no se aprobó de manera definitiva y por tanto, el documento careció de autoridad legal para dirigir la expansión urbana local. A pesar de ello, algunas partes del plano actual son una clara herencia de ese proyecto, ${ }^{27}$ aunque su materialización, realizada en época más reciente, presenta diferencias respecto a las líneas marcadas por el ingeniero burgalés.

El crecimiento espacial de Llanes se dirigió durante buena parte de la segunda mitad del siglo XX hacia el Noroeste de la villa, unos terrenos cuya ocupación, como hemos visto, ya se incluía en el proyecto de 1905. Aunque el trazado de determinados viales, como la calle Alfonso IX o la Avenida de las Gaviotas se ajusta fielmente a la trama ideada por Alberto Corral, el tejido urbano entre el Paseo de San Pedro y la carretera de Oviedo presenta, en su mayor parte, una mayor irregularidad y escasas coincidencias con el mencionado proyecto. Esta notable alteración respecto a la ortogonalidad propuesta en origen es resultado del largo proceso de urbanización de la zona, realizada bajo el influjo de otros proyectos posteriores y ejecutado después de que algunos solares hubiesen sido ocupados por algunos equipamientos de grandes dimensiones que implicaron una modificación del tamaño de las manzanas primigenias, pensadas para acoger únicamente un uso residencial.

Por lo que respecta a los ensanches de San Antón y La Portilla, si bien estos espacios forman parte hoy en día del suelo urbano de la villa, no se aprecian similitudes en el modo en el que se ocuparon respecto a lo aquí estudiado. Menor relevancia en el plano actual tienen, si cabe, las medidas propuestas para la reforma interior, puesto que la trama urbana del casco histórico se ha mantenido inalterada en su mayoría y las grandes actuaciones encaminadas a reformar el barrio del Cueto y a ampliar varias plazas, entre otras, no pasaron del papel.

27 Rubin (1993) señala algunas de las herencias del plano realizado por Alberto Corral en el Llanes de principios de los años 90, aunque en determinados casos diferimos con el mencionado autor. Así ocurre con la calle Nueva o vía Blanco, cuya apertura fue en realidad anterior a la redacción del proyecto. 


\section{Bibliografía}

Archivo Histórico Municipal de Llanes (AHMLL). Caja 467, expediente 467-2. Proyecto de reforma y ensanche de la villa de Llanes.

Archivo Histórico Municipal de Llanes (AHMLL). Caja 467, expediente 467-13. Memorias correspondientes al plano de la villa de Llanes.

Archivo Histórico Municipal de Llanes (AHMLL). Libros de actas del concejo, n. ${ }^{\circ}$ 29, 30, 32, 36, 37,38 y 39.

Archivo Histórico Municipal de Llanes (AHMLL). Plano de la villa de Llanes levantando por el Ingeniero de Caminos, Canales y Puertos D. Alberto Corral. Año 1902.

Archivo Municipal de Ribadedeva. Fondo Remigio Fernández. Plano general de Llanes. Mayo 1905.

Bassols Coma, M. (1973). Génesis y evolución del derecho urbanístico español (1812-1956). Madrid: Montecorvo.

Canella Secades, F. (1994). Historia de Llanes y su concejo (Edición facsimilar). Gijón: Mases.

Capel Sáez, H. (1975). Capitalismo y morfología urbana en España. Barcelona: Los Libros de la Frontera.

Capel Sáez, H. (2002). La morfología de las ciudades, vol. I. Barcelona: Ediciones del Serbal.

De Solá-Morales, M. (1997). Las formas de crecimiento urbano. Barcelona: Ediciones Universidad Politécnica de Cataluña.

Delgado Viñas, C. (1995). Las pequeñas y medianas capitales de provincia en el proceso de modernización del sistema urbano español. Las Palmas: Universidad de Las Palmas de Gran Canaria, Servicio de Publicaciones.

Esteban i Noguera, J. (1976). Los ensanches menores en la región de Barcelona (II). Barcelona: Laboratori d'urbanisme de Barcelona.

Estébanez Álvarez, J. (1989). Las ciudades: morfología y estructura. Madrid: Síntesis.

Fernández Cuesta, G. (2010). La ciudad burguesa: ciudades grandes, ciudades de tamaño medio, ciudades pequeñas y ensanches menores. En G. Fernández Cuesta y F. Quirós Linares (Dirs.), Atlas temático de España, vol. 1 (pp. 86-255). Oviedo, Ediciones Nobel.

Fernández Cuesta, G. (2012). San Sebastián: un modelo de construcción de la ciudad burguesa en España. Ería, 88, 101-128. 
Fernández Cuesta, G. (2015). Urbe II: la construcción histórica de la ciudad de Gijón. Oviedo: Ediciones de la Universidad de Oviedo.

Fernández García, A. (1980). Langreo, industria, población y desarrollo urbano. Oviedo: Universidad de Oviedo, Facultad de Letras.

López Trigal, L. (ed.) (1999). Los ensanches en el urbanismo español: el caso de León. Madrid: Biblioteca Nueva.

Llavona Campo, M. (2007). Una arquitectura de distinción: análisis y evolución de la casa indiana en el concejo de Llanes entre 1870 y 1936. Oviedo: Real Instituto de Estudios Asturianos.

Ministerio de Educación, Cultua y Deporte. Red Digital de Colecciones de Museos de España [Archivo fotográfico]. Recuperado de http://ceres.mcu.es

Morales Saro, M. C., y Bermejo, C. (Colabs.) (1993). Llanes, fin del siglo XIX: una contribución a los 125 años del periódico El Oriente de Asturias. Llanes: El Oriente de Asturias.

Morales Saro, M. C. (1999). Llanes y América: Cultura, arte y sociedad. México: Porrúa.

Murcia Navarro, E. (1981). Las villas costeras en el sistema urbano asturiano. Gijón: Silverio Cañada.

Quirós Linares, F. (1985). El proyecto de ensanche de Pola de Siero de 1932 en Ástura. Nuevos Cartafueyos d'Asturies, 3, 95-96.

Quirós Linares, F., y Tomé Fernández, S. (2001). El proceso de urbanización. Las ciudades. En Gil Olcina, A., y Gómez Mendoza, J. (eds.) Geografía de España (pp. 251-274). Barcelona: Ariel. Rubin de Celis, C. (1993). Un plano de Llanes de 1905. El Oriente de Asturias, núm. esp. 125 aniversario, mayo.

Sazatornil Ruiz, L. (1996). Arquitectura y desarrollo urbano de Cantabria en el siglo XIX. Santander: Universidad de Cantabria, Servicio de Publicaciones.

De Terán, F. (1999). Historia del urbanismo en España III. Siglos XIX y XX. Madrid: Cátedra.

Uría Maqua, J. (2004). La actividad marinera y mercantil de la villa de Llanes en el siglo XVI. En M. C. Morales Saro (Coord.), Llanes: viejas historias, nuevos patrimonios (pp. 67-106). Llanes: El Oriente de Asturias. 